\title{
Review: warfarin prevents stroke in non-rheumatic atrial fibrillation but has a higher risk for haemorrhage than other agents
}

Segal JB, McNamara RL, Miller MR, et al. Anticoagulants or antiplatelet therapy for non-rheumatic atrial fibrillation and flutter. Cochrane Database Syst Rev 2001;(1):CD001938 (latest version 29 Nov 2000).

\section{QUESTION: In patients with non-rheumatic atrial fibrillation (AF), how effective are anticoagulants and antiplatelet drugs in preventing thromboembolic complications?}

\section{Data sources}

Studies were identified by searching 3 databases and relevant journals.

\section{Study selection}

Studies were selected if they were randomised controlled trials (RCTs) evaluating warfarin, aspirin, combined low dose warfarin and aspirin, low molecular weight heparin, or indobufen for prevention of thromboembolism in AF or atrial flutter and if the outcomes were stroke, major bleeding, or death.

\section{Data extraction}

Data were extracted on study quality; patient characteristics and inclusion and exclusion criteria; therapeutic protocol, including intensity of coagulation and percentage of time inside and outside of the target range; and results.

\section{Main results}

14 RCTs were included. 6 RCTs compared warfarin with placebo: warfarin reduced the risk for stroke but increased the risk for major haemorrhage; the 23\% relative reduction in total mortality was not statistically significant. 4 RCTs compared aspirin with placebo: the modest reduction in stroke ( $16 \%)$ was not significant; no difference was seen for haemorrhage or mortality. 5 RCTs compared warfarin with all antiplatelet drugs: warfarin reduced the risk for stroke and showed no difference for haemorrhage or mortality. 4 RCTs compared warfarin with aspirin: a moderate decrease for stroke was shown with warfarin, and no difference was shown for haemorrhage or mortality. The table gives the results for stroke and haemorrhage. Adjusted dose war-
Source of funding: Agency for Healthcare Research and Quality.

For correspondence: DrJ Segal, John Hopkins School of

Medicine, 720 Rutland Avenue, Ross Building \#1025, Baltimore, $M D$ 21205, USA. farin reduced stroke more than low dose warfarin plus aspirin (2 RCTs). No differences were seen in comparisons of warfarin and indobufen (1 RCT) or low molecular weight heparin and placebo (1 RCT).

\section{Conclusions}

In patients with non-rheumatic atrial fibrillation, warfarin prevents stroke among those with average or higher risk and is more effective than antiplatelet drugs. The risk for major haemorrhage is increased with warfarin.

\section{COMMENTARY}

Despite convincing evidence that warfarin treatment is highly effective in preventing stroke in patients with chronic $\mathrm{AF}$, recent studies indicate that only one half of eligible patients are receiving this treatment. ${ }^{1}$ The most commonly cited reasons for warfarin underusage are concerns about bleeding in elderly patients and the feasibility of long term anticoagulation monitoring. ${ }^{2}$

For clinicians who remain unsure about the risk benefit ratio of warfarin treatment in patients with chronic $\mathrm{AF}$, the meta-analysis by Segal et al provides a concise balance sheet of potential benefits and harms associated with warfarin and aspirin treatment. For warfarin treatment, 18 patients need to be treated to prevent 1 additional stroke (RRR 66\%), whereas 1 additional major bleed will occur after the treatment of 77 patients. This risk benefit ratio is acceptable because about $70 \%$ of cardioembolic strokes are fatal or are associated with major disability. ${ }^{3}$ About $5 \%$ of warfarin associated bleeding episodes are intracranial, and most other bleeds are not associated with long term morbidity. ${ }^{4}$ Aspirin treatment is not associated with an increased risk for major bleeding. It has questionable efficacy, however, in overall stroke prevention (RRR 16\%), and its benefit may be limited to the prevention of thrombotic stroke.

Another consideration in deciding about treatment options is a patient's risk profile. Patients with previous stroke, left ventricular dysfunction, age $>75$ years, and hypertension are at high risk for stroke, whereas those with diabetes, coronary artery disease, and age $65-75$ years are at moderate risk, and those $<65$ years with no risk factors are at low risk. Patients $>75$ years of age are at the highest risk for bleeding, but will benefit most from treatment with warfarin.

In summary, warfarin should be considered in all patients with chronic $\mathrm{AF}$, and aspirin should be used only in patients with a contraindication to warfarin, or those at low risk of stroke.

James D Douketis, MD

St.Joseph's Hospital, Hamilton, Ontario, Canada

1 Smith NL, Psaty BM, Furberg CD, et al. Arch Intern Med 1999;159:1574-8.

2 Kutner M, Nixon G, Silverstone F. Arch Intern Med 1991; 151:1950-3.

3 Lin HJ, Wolf PA, Kelley-Hayes M, et al. Stroke 1996;27:1760-4. 4 Palareti G, Leali N, Coccheri S, et al. Lancet 1996;348:423-8. 